\title{
Relation of pyloric motility to pyloric opening and closure in healthy subjects
}

\author{
G Tougas, M Anvari, J Dent, S Somers, D Richards, G W Stevenson
}

\begin{abstract}
The relation between pyloric motor activity, opening, and closure was examined in eight healthy men. Manometry was performed with an assembly combining 13 side holes and a sleeve sensor positioned astride the pylorus. Simultaneous with manometry, pyloric opening and closure and antroduodenal contractions were observed fluoroscopically with the antrum filled with barium. During intraduodenal normal saline infusion, coordinated antral pressure waves swept over the pylorus and ejected barium into the duodenum. No localised pyloric motor pattern was observed under these conditions. In contrast, the intraduodenal triglyceride infusion was associated with the absence of antral pressure waves and virtual absence of antral wall movement. At the pylorus, there was a zone of luminal occlusion less than $1 \mathrm{~cm}$ long that persisted for the period of observation. This zone of luminal occlusion corresponded precisely with manometric recordings of a narrow zone of pyloric phasic and tonic activity. During the duodenal triglyceride infusion, the pylorus was closed for $\mathbf{9 8 . 5 \%}$ of the measurement period when basal pyloric pressure was $4 \mathrm{~mm} \mathrm{Hg}$ or more, and during this motor pattern, barium did not traverse the pylorus. Localised pyloric contractions cause sustained pyloric closure, whether these contractions are phasic or tonic. These contractions occur independently of antral or duodenal contractions and may interrupt gastric emptying.
\end{abstract}

Intraduodenal infusion of triglycerides and fatty acids delays gastric emptying in both humans and dogs. ${ }^{12}$ This effect has been ascribed to various demonstrated changes in motor function - suppression of antral contractions, ${ }^{3-5}$ reduction in gastric fundal tone ${ }^{6}$ and increased resistance to liquid gastroduodenal outflow. ${ }^{3}$ The exact role of the pylorus in controlling gastric outflow has been controversial for years. There are conflicting data on patterns of pyloric motility during fasting ${ }^{7-10}$ and during delivery of acid or lipids into the duodenum. Some studies in humans have shown stimulation of localised pyloric tonic and phasic contractions while others have shown no such responses. ${ }^{811} 12$

It has recently become evident that the conflicting findings on pyloric motor function have arisen primarily from the use of techniques that are poorly suited to pyloric manometry..$^{13} \mathrm{~A}$ detailed manometric side hole analysis of patterns of pyloric motility has shown that the pylorus contains a zone less than $1 \mathrm{~cm}$ in length which develops tonic and phasic contractions in response to intraduodenal infusions of tri- glyceride in association with suppression of antral pressure waves. ${ }^{13}$ The pyloric phasic contractions usually occur at a rate of about $3 /$ minute and have been named isolated pyloric pressure waves (IPPWs). Analysis of the mechanical patterns of the triglyceride induced pyloric motor response indicates that both the tonic and phasic components should be obstructive to flow of gastric contents into the duodenum.

In this study we have used a recently validated approach to pyloric manometry to investigate, in humans, the hypothesis that the pylorus has the capacity to interrupt gastric emptying. In order to do this, we stimulated localised pyloric tonic and phasic contractions with intraduodenal triglyceride infusion and observed fluoroscopically the effect of these contractions on pyloric opening and closure and the movement of barium from the stomach into the duodenum.

\section{Methods}

\section{SUBJECTS}

Studies were done in 11 healthy male volunteers whose mean age was 26 years (range 19-42 years). None reported taking any medications or drugs or had a past history of any gastrointestinal diseases or smoking. Written informed consent was obtained from each volunteer and the study was approved by the Ethical Committee and Research Advisory Committee of the McMaster University Medical Centre.

\section{EXPERIMENTAL PROTOCOL}

After a 12 hour overnight fast, a sleeve/side hole manometric assembly (see below) was passed through a nostril into the stomach. The subject then lay on his right side to help the passage of the weighted tip of the assembly into the duodenum.

The sleeve was then positioned so that it was astride the pylorus and the tip of the assembly was within the distal duodenum. Proper positioning of the assembly was assessed by three methods as follows: (1) progression of the distal end was observed by recognition of the usual fasting manometric patterns; (2) the location of the sleeve sensor was determined fluoroscopically before starting the experimental protocol, the sleeve having a metal coil built in along its length as a stiffener; (3) the exact location of the sleeve sensor across the pylorus was confirmed and subsequently monitored using continuous dual point transmucosal potential difference measurements (TMPD) (see below).

After positioning of the sleeve sensor astride the pylorus, and after the occurrence of the first episode of phase III of the interdigestive migrat-

ing motor complex, normal saline solution was
Correspondence to: Dr G Tougas, McMaster Room 4W8, 1200 Main Stree West, Hamilton, Ontario Accepted for publication 10 June 1991 
infused at $1 \mathrm{ml} / \mathrm{minute}$ (four subjects) or $3 \mathrm{ml} /$ minute (four subjects) into the duodenum for five minutes. The infusate entered the duodenum 5 $\mathrm{cm}$ distal to the aborad margin of the sleeve sensor via a channel incorporated into the manometric assembly. A 50 to $100 \mathrm{ml}$ bolus of a diluted barium sulphate suspension was then swallowed, the transpyloric flow of which was observed fluoroscopically for the subsequent three minutes with the subject lying on his right side in the position that displayed the pylorus best (see below).

After a 10 minute recovery period, and with the subject still lying on his right side, a triglyceride emulsion (Intralipid 10\%) was infused into the duodenum, again at rates of 1 to $3 \mathrm{ml} /$ minute (four subjects at each rate) for five minutes. The volunteer then took a further 100 cc of barium and the distal antral and pyloric outlines were examined fluoroscopically for three minutes, during which manometric recordings were made and the triglyceride infusion maintained at the same rate.

The Intralipid 10\% (Kabivitrum, Dorval, Canada) is a fractionated soya bean emulsion in water with $10 \mathrm{~g}$ of soya bean oil, $1.2 \mathrm{~g}$ of egg phospholipid, $2 \cdot 25 \mathrm{~g}$ of glycerine $/ 100 \mathrm{ml}$. Its osmolarity is $280 \mathrm{mOsm} / 1$, and its caloric content is $1100 \mathrm{kcal} / \mathrm{l}(4620 \mathrm{~kJ} / \mathrm{l})$. Its major fatty acids are linoleic acid and oleic acid.

\section{MANOMETRIC TECHNIQUE}

The methods of antropyloroduodenal manometry used in this study have been described in detail previously..$^{14}$ A 14 lumen manometric sleeve assembly was built specifically for these experiments. The $4.5 \mathrm{~cm}$ long sleeve was used to monitor pyloric pressures. ${ }^{15}$ The arrangement of side holes is shown in Figure 1. The side holes located at the upper margin of the sleeve, and at 2.5 and $5 \mathrm{~cm}$ orad to this level, monitored antral pressures. The side holes arrayed at $5 \mathrm{~mm}$ intervals within the sleeve length allowed assessment of the spatial patterns of pressure waves across the pylorus. Duodenal pressures were monitored from the three side holes $0,2 \cdot 5$, and 5 $\mathrm{cm}$ aborad from the distal sleeve end. The most distal duodenal side hole was used also to infuse the saline or triglyceride emulsion into the duodenum. A tungsten weight was incorporated into the distal end of the assembly to aid its passage from the stomach into the duodenum.

The side holes and sleeve sensor were perfused by two low compliance pneumohydraulic perfusion systems (Arndorfer Medical Specialties, Greendale, Wisconsin, USA, and Mui Scientific, Mississauga, Ontario, Canada). The reservoirs of both pumps were set at a pressure of $15 \mathrm{psi}$ giving a flow of $0.6 \mathrm{ml} / \mathrm{min}$ to each manometric channel. Degassed distilled water was used in all channels except the two side holes at the orad and aborad sleeve margins. These were perfused with normal saline from separate reservoirs so measurements of TMPD could be made concurrently with manometry.

Pressures were detected with external transducers - either model 4-327-1 (Bell and Howell Ltd, Pasadena, California, USA) or model 12N4696 (Cobe Ltd, Lakewood, Colorado, USA) - on two eight channel polygraphs, these being a Grass model 17D (Quincy, Massachussetts, USA) and a Sensormedics Dynograph 611 (Schiller Park, Illinois, USA).

\section{TRANSMUCOSAL POTENTIAL DIFFERENCE} MEASUREMENTS

Transmucosal potential difference was detected from the saline perfused side holes at both ends of the sleeve using previously described methods. ${ }^{13}$ The saline perfusate transmitted the potential up the manometric assembly into the transducer dome from which a bridge containing $1 \mathrm{~mol} / \mathrm{l}$ potassium chloride in 3\% agar transferred the signal to a calomel half cell (Ionode, Brisbane, Australia). A saline filled subcutaneous electrode inserted into the forearm served as a reference electrode. The potentials were detected with a dual channel purpose built system which gave a continuous output to the Grass recorder (Multichannel $\mathrm{p} / \mathrm{pH}$ system 1201, BME, Flinders Medical Centre, Bedford Park, South Australia, Australia).

The position of the sleeve was adjusted so that the following criteria for position were satisfied: (1) duodenal side hole TMPD equal to or more negative than $-15 \mathrm{mV}$; (2) gastric side hole TMPD equal to or more positive than $-20 \mathrm{mV}$; and (3) a gradient between the two TMPD side holes of greater than $15 \mathrm{mV}$.

\section{RADIOLOGY}

Fluoroscopy was done on a remote control table. Before manometric recordings were started, the optimal position for radiological evaluation of the pylorus was determined by giving the subject a small volume of barium sulphate. The subject was then positioned so that a right angled view of the pyloric canal was obtained, to permit evaluation of pyloric canal diameter and determine whether the pylorus was open or closed. This usually required a prone oblique position, occasionally supine oblique, both with the right side down, and with variable table tilt of up to $50^{\circ}$. This position was maintained thereafter. A small field of view was used, approximately $6 \times 6$ 
$\mathrm{cm}$, and gonads as well as the neck were shielded in all studies.

The radiation dosage was calculated using a $6 \times 6$ skin entry exposure, with the patient oblique, so that the patient's spinal cord was not in the primary beam. Using these parameters, the estimated radiation dose was $22-25 \mathrm{mrad}$ $\left(22-25 \times 10^{-5} \mathrm{~Gy}\right)$.

The fluoroscopy was recorded on a $3 / 4$ inch video cassette recorder (Sony) with simultaneous recording of a superimposed image of the sleeve manometric tracing processed through a signal mixer (Hewlett-Packard). This allowed for exact temporal correlation of the radiological data with the pyloric manometric recordings.

\section{ANALYSIS OF DATA}

\section{Manometry}

Records were only analysed when the TMPD readings satisfied the criteria for correct sleeve position as given above.

Pressure waves were defined as increases in intraluminal pressure equal to or greater than 10 $\mathrm{mm} \mathrm{Hg}$ which lasted less than 20 seconds.

When the sleeve recording indicated a pressure wave which occurred in the absence of any discernible deflection of antral or duodenal pressure as recorded by the side holes at or beyond the margins of the sleeve, an isolated pyloric pressure wave (IPPW) was scored. When there was an antral or duodenal pressure wave that had occurred within 2 seconds of the onset of the IPPW recorded by the sleeve, an antropyloric or antropyloroduodenal wave was scored.

When an IPPW was scored, its longitudinal extent and position on the sleeve was defined by examination of the tracings from the array of side holes at $5 \mathrm{~mm}$ intervals along the sleeve length (Fig 1).

Basal pyloric pressure obtained with the sleeve sensor was referenced to basal antral pressure. For the purposes of analysis of basal pyloric pressure, IPPWs were edited by drawing a straight line from the onset of their major upstroke to the end of their major downstroke. Mean basal pyloric pressure was expressed as the mean values for each 15 second period.

Manometric responses

\section{Radiology}

The state of the pyloric lumen was defined as open, closed, or indeterminate for each of the 15 second periods in which mean basal pyloric pressure was measured. If the lumen was open at any time during the 15 seconds, the period was defined as open. In general it was possible to determine precisely the moment that the pylorus opened and closed. The state of the pylorus was indeterminate only when the pyloric canal was obscured by barium containing overlapping loops of distal duodenum or proximal duodenum or jejunum, despite best attempts at achievement of optimal positioning of the $x$ ray tube and subject.

\section{Statistical analysis}

A $2 \times 2$ table was constructed for correlation of pyloric opening or closure versus pyloric pressure (relative to antral pressure) and one way analysis of variance (Anova) was used for statistical analysis of the relation between pyloric tone and opening of the pyloric lumen.

The paired Student's $t$ test was used for statistical validation of the response to intraduodenal lipid infusion compared with saline infusion.

\section{Results}

Studies were performed in 11 subjects, but incomplete data were obtained in three early on in the project because the introduction of the barium into the stomach resulted in displacement of the sleeve into the antrum which could not be rectified by attempts at repositioning. Subsequently, this aborad movement of the pylorus was allowed for by passing the sleeve distally into the duodenum before giving barium. The data presented below are derived solely from the eight subjects in whom technically satisfactory recordings were obtained under the two study conditions.

The patterns of motility associated with intraduodenal triglyceride infusion were as described previously, ${ }^{1+}$ being pyloric tone, IPPWs, and suppression of antral and duodenal contractions (Fig 2). By contrast, the dominant motor pattern associated with intraduodenal saline infusion was
Figure 2: Manometric tracings showing the patterns of antropyloroduodenal motility during duodenal saline and triglyceride infusions. With saline infusion, antral pressure waves are propagated into the pylorus whereas during triglyceride infusion, pyloric tonic activity and isolated pyloric pressure waves are present and antral activity is absent.
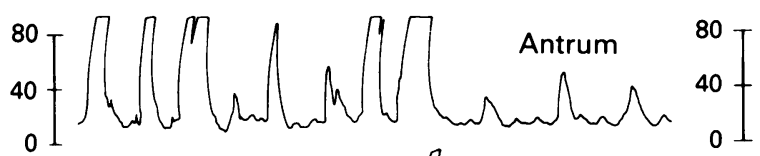

Antrum
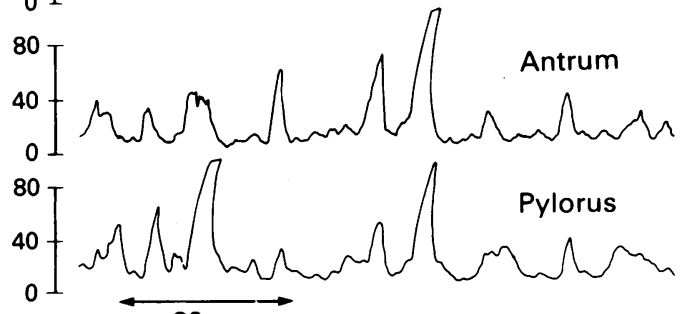

$30 \mathrm{~s}$
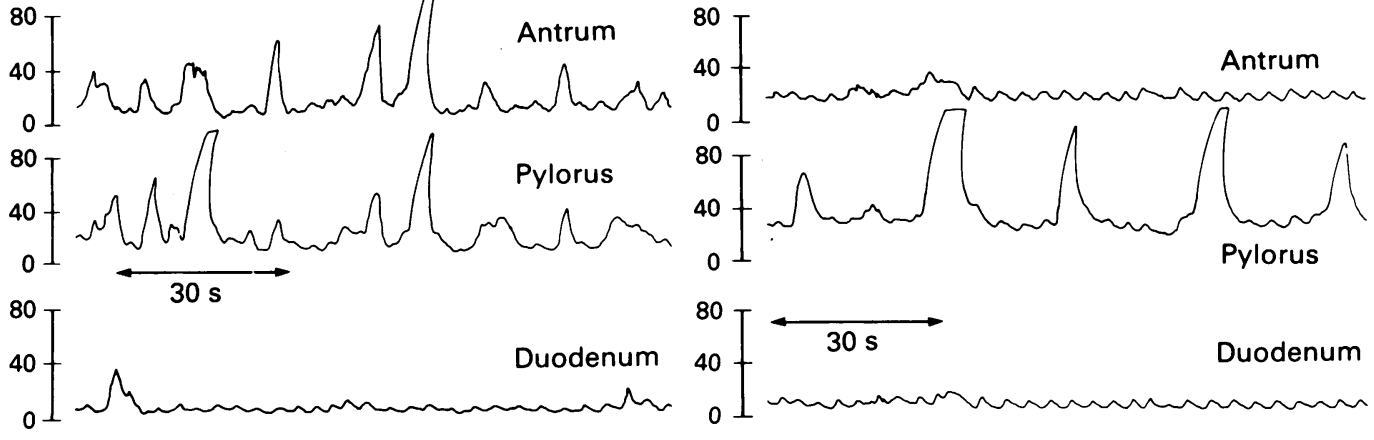

Intraduodenal saline

Intraduodenal triglyceride 
TABLE I Pyloric manometric patterns during normal saline or triglyceride duodenal infusion

\begin{tabular}{llll}
\hline \multicolumn{4}{l}{ Duodenal infusate } \\
\cline { 2 - 4 } & Saline & Triglycerids & $p$ \\
\hline Pyloric pressure (mm & $0.33(2.04)$ & $6.56(3.63)$ & $<0.0001$ \\
$\quad$ Hg) & $0.97(0.39)$ & $2.63(0.73)$ & $<0.002$ \\
IPPWs (no/min) & 0.00 \\
\hline
\end{tabular}

IPPW $=$ isolated pyloric pressure waves.

Values, mean (SD).

TABLE II Relation between pyloric aperture and pyloric pressure

\begin{tabular}{llc}
\hline & \multicolumn{2}{c}{ Pyloric lumen } \\
\cline { 2 - 3 } Pyloric pressure & Closed & Open \\
\hline$>2 \mathrm{~mm} \mathrm{Hg}$ & $1(1 \cdot 6 \%)$ & $63(98 \cdot 4 \%)$ \\
$\leqslant 2 \mathrm{~mm} \mathrm{Hg}$ & $72(97 \cdot 4 \%)$ & $2(2 \cdot 6 \%)$ \\
\hline
\end{tabular}

The number is the total of 15 second periods evaluated.

irregular occurrence of antropyloroduodenal pressure waves, and absence of pyloric tone and IPPWs (Fig 2). Data on the patterns of pyloric motility that occurred during the period of fluoroscopic observation in the eight subjects are given in Table I.

Table II summarises the relation of sleeve recorded basal pyloric pressure to the presence of a barium column across the pylorus for the two conditions studied. In $98.4 \%$ of the 15 second periods during which basal pressure was greater than $2 \mathrm{~mm} \mathrm{Hg}$, the pylorus was closed around the sleeve assembly. A barium column extended across the pylorus in $97.4 \%$ of periods in which basal pyloric pressure was less than or equal to 2 $\mathrm{mm} \mathrm{Hg}$. Pyloric pressure was present intermittently in two subjects; pyloric closure and opening were consistently related to the presence or absence of pyloric pressure greater than $2 \mathrm{~mm}$ $\mathrm{Hg}$.

All IPPWs occurred when pyloric tone was present. Consequently, the pylorus was always closed just before the onset of each IPPW. The presence of tone made it impossible to examine the impact of IPPWs alone on patterns of pyloric opening and closure. Throughout every IPPW, however, pyloric closure was maintained, there the sleeve on the bottom
right, isolated pyloric pressure waves are present in the pyloric segment. being no evidence of peristaltic pumping of barium by this type of contraction.

Antropyloroduodenal waves occurred in the setting of absent pyloric tone in 7 of the 8 subjects. These waves occluded the lumen of the distal antrum as they advanced towards the already open pylorus. Pumping of barium was observed in advance of the lumen occluding contraction. The pylorus took part in the progression of the wave into the duodenum as the pressure wave and radiological zone of lumen occlusion were seen to traverse the side holes arrayed along the sleeve length. The six antropyloroduodenal waves that occurred in the subject in whom pyloric tone was present in association with saline infusion also propelled barium across the pylorus.

The radiological outlines of the antropyloroduodenal segment were distinctly different during the two study conditions. In association with triglyceride infusion, the antrum was globular and because there was no emptying of barium into the duodenum, it was not necessary to refill the antrum with barium as recordings were made. There was a deep, static, lumen occluding ring at the pylorus when pyloric tone and IPPWs were present. This ring was approximately $1 \mathrm{~cm}$ long as judged by comparison with the length of the spring wire which stiffened the sleeve (Fig 3). This ring was always seen to be astride the sleeve length when the TMPD recordings satisfied criteria for correct positioning (see methods). The appearance of the ring did not change during IPPWs, indicating that tonic and phasic contractions occurred in an identical zone. The length of the pyloric lumen that was observed to be consistently occluded correlated well with manometry, as $89 \%(61 / 65)$ of all IPPWs recorded during fluoroscopy were seen in only one side hole indicating that the zone of localised pyloric contraction was usually equal to or less than $1 \mathrm{~cm}$ in length. In keeping with the radiological appearances, pyloric tone was recorded from the same side holes as IPPWs. The position of the side hole that recorded pyloric tone and IPPWs was in accord with the position of the lumen occluding ring on the pylorus, as judged fluoroscopically by reference to the sleeve spring wire stiffener.

In two subjects there was no discernible indentation of the antrum that was either temporally linked or independent of IPPWs. In the other six subjects, a detectable ripple of the antral contour which propagated aborally, but died out before it reached the terminal antrum was seen. The antral ripple was not accompanied by any increase in antral pressure nor was it clearly coordinated with IPPWs (Fig 4).

In association with intraduodenal saline infusion, the antrum emptied rapidly and so required refilling with barium during the period of fluoroscopy. No discrete pyloric ring could be discerned (Fig 5).

\section{Discussion}

These studies are the first performed in humans in which it has been possible to examine systematically the effect of pyloric tone and IPPWs on transpyloric flow. The observations illustrate 


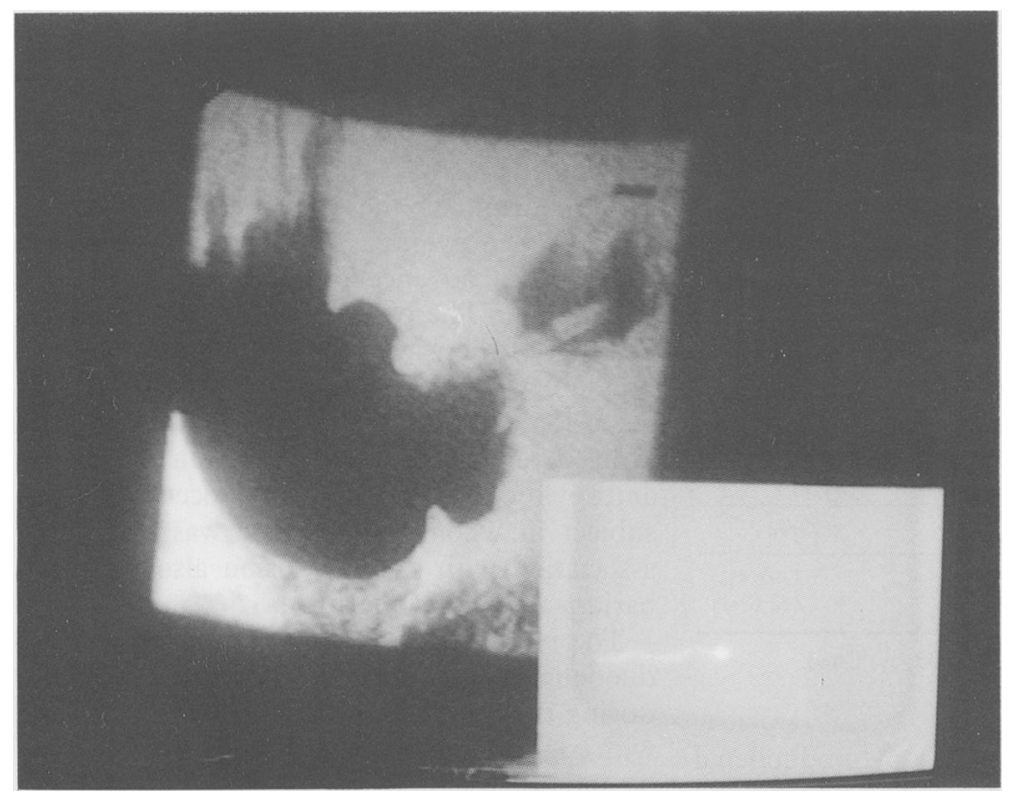

Figure 4: Antral 'ripple' of typical amplitude seen during duodenal triglyceride infusion which was causing isolated pyloric pressure waves (IPPWs) and pyloric tone. As seen in the motility tracing, the ripple was not related in time to an IPPW and did not produce any antral luminal pressure increase. The IPPWs that occurred during the same period were not phase locked with these antral ripples.

Figure 5: Fluoroscopic appearance of the pylorus during intraduodenal saline infusion. The pylorus is wide open and barium is seen within the lumen. No pyloric tone or isolated pyloric pressure waves were occurring, the oscillations of the manometer were caused by respiration opening and closure in humans. We used fluoroscopy, since this is the only technique that gives adequate resolution of the luminal contours on a second by second basis. The manometric technique used has been developed specifically for pyloric manometry and has been validated recently by us. ${ }^{13}$ Our data in this study indicate clearly that a basal pyloric pressure more than 2 $\mathrm{mm} \mathrm{Hg}$ above distal antral pressure occludes the human pyloric lumen and consequently prevents transpyloric flow, since the associated suppression of antral contractions means that there are no antral forces that can overcome this weak luminal occlusion. Our observations in this study now define the functional significance of pyloric motor responses that we have recorded manometrically in previous human studies. ${ }^{13}$ is

Studies of the pyloric diameter in conscious dogs by Ehrlein have produced results consistent with those reported in the present study. ${ }^{17}$ Ehrlein used the extraluminal inductograph method originally developed by Brody and Quigley. ${ }^{18}$ Duodenal instillation of hydrochloric acid or oleic acid resulted in a diminution of the pyloric diameter to a level consistent with luminal closure, a change that was associated with the cessation of gastric emptying. ${ }^{17}$ This supports the view that pyloric closure plays a role in preventing transpyloric flow. The methods used by Ehrlein would not have allowed him to recognise phasic pyloric contractions if the pylorus were already closed. Thus, the tracings obtained by Ehrlein in his study are consistent with our own data.

What is the mechanical importance of IPPWs? The present study suggests that they are relatively unimportant as controllers of transpyloric flow, given that pyloric tone was always present when IPPWs occurred and that pyloric tone alone closed the pylorus. In our previous studies, however, we have often recorded stimulation of IPPWs in the absence of pyloric tone ${ }^{14}$ and we expected that this would be the case in the present study. Our fluoroscopic and manometric data indicate that IPPWs are non-propagated contractions localised to the zone of tonically contracted pylorus. The use of multiple manometric sampling points and simultaneous fluoroscopy shows clearly that IPPWs are distinct from the terminal antral contractions observed in humans by Smith et al. ${ }^{19} \mathrm{We}$ propose that transpyloric flow would also be obstructed during IPPWs that occur in the absence of pyloric tone, the effect of this being to 'chop' any transpyloric flow that might be occurring between IPPWs. Such an effect could produce a more graded braking of gastric emptying by the pylorus than could be produced by a pyloric tonic response. It is possible that IPPW responses occur without the development of pyloric tone at lower levels of stimulation of duodenal receptors by nutrients. ${ }^{\text {s }}$ The high rate of intraduodenal lipid infusion used in the present study may explain why we saw pyloric tone so consistently, since triglyceride was delivered to the duodenum at a rate twice that used in a previous study in which we recorded stimulation of IPPWs without tone in about half of the subjects. Furthermore, after food we have recorded intermittent occurrence of IPPWs alone. ${ }^{20}$ 
The radiological appearances during saline and triglyceride infusions were markedly different, but consistent with the manometric data. Many would consider that the appearances associated with triglyceride infusion are unusual. Virtually all fluoroscopic studies of the pylorus have been made during fasting, a setting in which localised pyloric contractions do not normally occur. ${ }^{21}$ Consequently, radiological observations have contributed little to the concept that the pylorus may play a motor role that is independent of the antrum and duodenum. Our observations of the pylorus during delivery of triglyceride to the duodenum are not unique, since similar appearances were reported by Cannon in cats when cream was infused into the duodenum. ${ }^{22}$ White et al have reported very similar findings to our own in one healthy human volunteer when triglyceride rich barium was ingested. ${ }^{23}$

Although the method of our study did not allow us to measure transpyloric flow, it was readily apparent nevertheless that, usually when the pylorus was open, transpyloric flow was occurring and was especially rapid just before lumen occlusion by antropyloroduodenal contractions. Similarly, pyloric closure consistently prevented transpyloric flow. Demonstration of the capacity of the pylorus to suspend transpyloric flow does not establish the place, if any, that the pylorus has in the control of gastric emptying, but merely establishes that it is a mechanism that can indeed stop emptying. This mechanism should be considered as one among several that contribute to slowing of gastric emptying - the other mechanisms that are currently recognised being fundic relaxation, suppression of antral contractions, and stimulation of duodenal resistance to flow. During emptying of high calorie nutrient liquids and small nutrient solid-liquid meals, IPPWs occur interspersed with a mixture of coordinated contractions that involve the antrum, pylorus, and duodenum. ${ }^{20}$ These observations and the effect of pyloroplasty on gastric emptying of nutrients suggests that the pylorus has an important role in slowing of emptying. ${ }^{24} 25$ The insights into the mechanics of pyloric motility gained from the present study will allow systematic investigation of the settings in which the pylorus is of particular importance for the control of gastric emptying.

GT was supported by the CAG-Merck Research Fellowship, the RMA Scholarship, and the Canadian Medical Research Council.
JD was supported by the Canadian Medical Research Council and a grant from Astra Pharmaceuticals, Sydney, Australia. This work was presented in part at the Meeting of the American Gastroenterological Association in May 1988

1 Keinke O, Ehrlein H-J. Effect of oleic acid on canine gastroduodenal motility, pyloric diameter and gastric emptying. Quart $\mathcal{F}$ Exp Physiol 1983; 68: 675-86.

2 Shafer RB, Levine AS, Marlette JM, Morley JE. Do calories osmolality or calcium determine gastric emptying? Am $\mathcal{f}$ Physiol 1985; 248: R479-83.

3 Quigley JP, Zettleman JH, Ivy AC. Analysis of factors involved in gastric motor inhibition by fats. Am $\mathcal{F}$ Physiol 1934; 108: 643-51.

4 Weisbrodt NW, Wiley JN, Overholt BF, Bass P. A relation between gastroduodenal muscle contractions and gastric emptying. Gut 1969; 10: 543-8.

5 White CM, Poxon V, Alexander-Williams J. Effects of nutrient liquids on human gastroduodenal motor activity. Gut 1983; 24: 1109-16.

6 Dooley CP, Reznick JB, Valenzuela JE. Variations in gastric and duodenal motility during gastric emptying of liquid meals in humans. Gastroenterology 1984; 87:1114-9.

7 Fisher RS, Cohen S. Physiological characteristics of the human pyloric sphincter. Gastroenterology 1973; 64: 67-75.

8 Valenzuela JE, Defilippi C. Pyloric sphincter studies in peptic ulcer patients. Dig Dis 1976; 21 : 229-32.

9 Kaye MD, Mehta SJ, Showalter JP. Manometric studies of the human pylorus. Gastroenterology 1976; 70: 477-80.

10 McShane AJ, O'Morain C, Lennon JR, Coakley JB, Alton BG. Atraumatic non-distorting pyloric sphincter pressure stradies. Gut 1976; 21: 826-8.

11 Tougas G, Allescher H-D, Li YY, Daniel EE, Dent J, Hunt RH. Effect of duodenal acidification on pyloroduodenal motor activity in humans. Gastroenterology 1988; 94: A464.

12 White CM, Poxon V, Alexander-Williams. A study of motility of normal human gastroduodenal region. Dig Dis Sci 1981; 26: $609-17$.

13 Heddle R, Dent J, Toouli J, Read NW'. Topography and measurement of pyloric pressure waves and tone in humans. Am F Physiol 1988; 255: G490-97.

14 Heddle R, Dent J, Read NW, Houghton LA, Maddern GJ, Horowitz M, et al. Antropyloroduodenal motor responses to intraduodenal lipid infusion in healthy volunteers. $A m \mathcal{F}$ Physiol 1988; 254: G671-9.

15 Dent J. A new technique for continuous sphincter pressure measurement. Gastroenterology 1976; 71: 263-7.

16 Meyer JH. Motility of the stomach and the gastroduodenal junction. In: Johnson LR, ed. Physiology of the gastrointestinal tract (2nd ed). New York: Raven Press, 1987: 613-

17 Ehrlein H-J. Motility of the pyloric sphincter studied by the inductograph method in conscious dogs. Am $\mathcal{F}$ Physiol 1988; 254: G650-7.

18 Brody DA, Quigley JP. Application of the 'inductograph' to the registration of movements of body structures such as the pyloric sphincter. F Lab Clin Med 1944; 29: 863-7.

19 Smith AWM, Code CF, Schlegel JF. Simultaneous cineradiographic and kymographic studies of human gastric antral motility. F Appl Physiol 1957; 11: 12-16.

20 Houghton LA, Read NW, Heddle R, Horowitz M, Collins PJ, Chatterton B, et al. Relationship of the motor activity of the antrum, pylorus and duodenum to gastric emptying of a solid-liquid mixed meal. Gastroenterology 1988; 94 1285-91

21 Carlson HC, Code CF, Nelson RA. Motor action of the canine gastroduodenal junction: a cineradiographic, pressure, and electrical study. Am $\mathcal{F}$ Dig Dis 1966; 11: 155-76.

22 Cannon WB. The movements of the stomach studied by means of the Roentgen rays. Am $\mathcal{F}$ Physiol 1898; 1 : 359-82.

23 White CM, Poxon V, Alexander-Williams. The importance of the distal stomach in gastric emptying of liquids in man. Surg Gastroenterol 1984; 3: 13-20.

24 Muller-Lissner SA, Blum A. To-and-fro movements across the canine pylorus. Scand f Gastro $1984 ; 19$ (Suppl 92): 1-3.

25 Hinder RA, Bremmer CG. Relative role of pyloroplasty size, truncal vagotomy, and milk meal volume in canine gastric emptying. Dig Dis 1978; 23: 210-6. 